\title{
Juvenile myasthenia gravis: an unusual presentation
}

\author{
Phillips H. Winter and Charles F. Koopmann Jr. \\ Department of Otolaryngology, University of Michigan, Ann Arbor, MI 48109-0312 (U.S.A.) \\ (Received 5 June 1989) \\ (Revised version received 21 December 1989) \\ (Accepted 8 February 1990)
}

Key words: Myasthenia gravis; Stridor; Child

\begin{abstract}
Myasthenia gravis is a disease characterized by muscular weakness and fatigability which afflicts $2-10$ patients per 100,000 with $20 \%$ of cases presenting in childhood. Muscles innervated by cranial nerves are frequently involved with ptosis and diplopia being the most frequent presenting complaints. We review the literature and report a case of a 20 -month-old infant presenting with inspiratory stridor and cyanosis, resembling foreign body aspiration.
\end{abstract}

\section{Introduction}

Myasthenia gravis is a neuromuscular disorder characterized by muscular weakness and fatigability with partial or complete restoration of strength after rest or after administration of anticholinesterase drugs. Any muscle innervated by cranial nerves can be involved but ptosis is the most common presenting manifestation in children and adults [6]. We report a case of juvenile myasthenia gravis presenting as inspiratory stridor and cyanosis resembling foreign body aspiration.

\section{Case report}

C.H. was a 20-month-old white female who presented to the University of Michigan Medical Center Emergency Room with a 24-h history of inspiratory

Correspondence: P.H. Winter, Dept. of Otolaryngology, University of Michigan, Ann Arbor, MI, 48109-0312, U.S.A. 
stridor, change in voice and cough. In the hour preceding the onset of symptoms, the patient ingested peanuts and corn chips, but no definite history of foreign body aspiration was obtained. With the exception of an uncomplicated mild upper respiratory infection 5 days prior to admission, the child was in good health, and had no prior hospitalizations. She was the product of a term pregnancy and normal spontaneous vaginal delivery and had Apgar scores of $7 / 8$; mental and physical development was normal for her age. Her immunizations were current and she was on no medications. There was no family history of neuromuscular disorders.

Physical examination revealed an afebrile infant with a respiratory rate of 24 and pulse of 120 . She exhibited no stridor at rest but became cyanotic with inspiratory stridor when agitated. The remainder of her head and neck examination was normal. Her respiratory examination was normal with no wheezing or rhonchi at rest. Inspiratory and expiratory chest radiographs were normal.

Because of the severity of the patient's stridor and the possibility of foreign body aspiration, direct laryngoscopy and bronchoscopy was performed, however, no foreign bodies or anatomic abnormalities were noted in the aerodigestive tract or tracheobronchial tree. Her true vocal cords were seen to be in adduction while we passed the bronchoscope. Full abduction of her vocal cords was not demonstrated during endoscopy. The postoperative period was uneventful until $16 \mathrm{~h}$ later when she again developed inspiratory stridor and cyanosis with agitation. Examination revealed mild dysarthric speech, drooling and head lag. No stridor was noted at rest and baseline oxygen saturations were $95 \%$ but with stridor, oxygen saturations fell to $75 \%$. Shortly thereafter, she developed bilateral ptosis and dysconjugate gaze. Facial expression was initially normal but bilateral weakness developed later in the day. Gag reflex was intact and the remainder of the otolaryngologic and neurologic examination was normal.

Lumbar puncture revealed 1 RBC, 0 WBC's, protein of $13 \mathrm{mg} / \mathrm{dl}$ and glucose of $62 \mathrm{mg} / \mathrm{dl}$. Ammonia and toxicology screens were normal. A CT scan of the head was normal but while undergoing the scan, the patient became agitated with inspiratory stridor and cyanosis requiring intubation. The cyanosis resolved without mechanical ventilation. Neuromuscular junction transmission was tested by measuring the compound muscle action potential following repetitive stimulation of the motor nerve at 2-3 Hz. No decrement was recorded when stimulating the tibial, median, ulnar and spinal accessory nerves. A $48 \%$ decrement was observed stimulating the facial nerve. Postactivation repair of the decrement could not be assessed due to the need to sedate the patient. Concentric needle EMG study of a limb muscle revealed no abnormal spontaneous activity and motor unit potentials were normal. Her ptosis, diplopia and facial weakness reversed with the intravenous administration of edrophonium chloride (Tensilon) confirming the diagnosis of myasthenia gravis.

Further evaluation included normal chest CT scan, thyroid function tests, total hemolytic complement levels, and titers of antinuclear antibodies, rheumatoid factor, acetylcholine receptor antibodies, and striated muscle antibodies. Her symptoms resolved on oral prostigmine methyl sulfate (neostigmine) and she continued to be asymptomatic 19 months later. 


\section{Discussion}

Myasthenia gravis is an autoimmune disease with a prevalence of $2-10$ per 100,000 with $20 \%$ of the patients presenting before 20 years of age [2]. The diagnosis of myasthenia gravis rests on an appropriate history and the reversal of symptoms after the administration of anticholinesterase medications or the electrodiagnostic findings of a postsynaptic defect in neuromuscular transmission. When weakness is mild and the above diagnostic tests are inconclusive, measurement of neuromuscular junction transmission by single fiber techniques is the most sensitive test [5]. Abnormal titers of antibodies to the acetylcholine receptor are felt to be diagnostic of myasthenia gravis [1], but children with myasthenia gravis have a lower incidence of serum acetylcholine receptor antibodies which are normal in $40 \%$ of young patients [7].

Other conditions which mimic myasthenia gravis include botulism, LambertEaton myasthenic syndrome and congenital myasthenia gravis [2]. The lack of abnormal spontaneous activity on needle electromyography and prompt and complete response to edrophonium chloride do not support botulism. There was no electrodiagnostic evidence for a myasthenic syndrome because the amplitudes of the compound action potentials were normal and no facilitation was observed at $10 \mathrm{~Hz}$ stimulation. Congenital myasthenia gravis presents with weakness at birth or shortly thereafter and involvement of the extraocular muscles is the predominant feature with variable degrees of generalized weakness [2].

Myasthenia gravis has been divided into subtypes based on age at presentation and distribution of weakness. Juvenile myasthenia gravis is clinically similar to adult onset autoimmune myasthenia gravis [2]. In juvenile myasthenia gravis girls are more frequently afflicted than boys and ptosis with or without diplopia is the most frequent presenting complaint. Among all patients with juvenile myasthenia, 20\% will have complete resolution of symptoms; most commonly, those with only ocular findings have the best prognosis. An acute fulminant form of myasthenia has been described in children in which respiratory crisis is the initial feature of the disease with dyspnea and cyanosis developing over $24 \mathrm{~h}$, due to involvement of thoracic respiratory musculature [2].

Weakness of muscles innervated by cranial nerves is common in myasthenia gravis with ptosis and dysconjugate gaze most frequent [7]. Inspiratory stridor has not previously been reported as a presenting symptom in the pediatric population, and only two cases of laryngeal involvement have been found in adults. The first report was a 67-year-old man who presented with breathy dysphonia and dyspnea on exertion. Direct laryngoscopy in this patient revealed initially normal vocal cord mobility, but on repeated phonation abduction was not greater than 1-2 mm.

Rapid administration of intravenous Tensilon reversed the vocal cord hypomobility [4]. The second report is a 25-year-old man who developed inspiratory stridor after being overcome by fumes from a chlorine gas explosion. Direct laryngoscopy showed minimal vocal cord abduction with inspiration. This patient's inspiratory stridor was reversed with the administration of Tensilon [3]. The present report identifies an infant with myasthenia gravis presenting with inspiratory stridor and 
cyanosis resembling foreign body aspiration. Direct laryngoscopy revealed normal laryngeal and tracheobronchial anatomy and no foreign bodies were demonstrated. While normal mobility of this child's vocal cords was not demonstrated, adduction of the vocal folds was noted while attempting to pass the bronchoscope. Her initial postoperative course was uneventful and was without inspiratory stridor. When the child again developed inspiratory stridor $18 \mathrm{~h}$ later, she also developed ptosis, head lag, drooling and dysarthria which prompted the diagnostic Tensilon test.

\section{Conclusion}

In conclusion, we report a rare case of juvenile myasthenia gravis presenting with inspiratory stridor and cyanosis. Myasthenia gravis can present with involvement of any muscle innervated by cranial nerves and the diagnosis should be entertained when muscular weakness or fatigability is demonstrated.

\section{References}

1 Drachman, D.B., Myasthenia gravis, N. Engl. J. Med., 298 (1978) 136-142.

2 Fenichel, G.M., Clinical syndromes of myasthenia in infancy and childhood: a review, Arch. Neurol., 35 (1978) 97-103.

3 Foulks, C.J., Myasthenia gravis presenting as laryngeal stridor after exposure to chlorine gas, South Med. J., 74 (1981) 1423-1424.

4 Friedman, S. and Goffin, F.B., Abductor vocal weakness in myasthenia gravis: report of a case, Laryngoscope, 76 (1966) 1520-1523.

5 Sanders, D.B. and Howard, J.F., Single-fiber electromyography in myasthenia gravis, Muscle Nerve, 9 (1986) 809-819.

6 Seybold, M.E., Myasthenia gravis: a clinical and basic science review, J. Am. Med. Assoc., 250 (1983) 2516-2521.

7 Snead, O.C., Benton, J.W., Dwyer, D., Morley, B.J., Kemp, G.E., Bradley, R.J. and Oh, S.J., Juvenile myasthenia gravis, Neurology, 30 (1980) 732-739. 